\title{
Accuracy of Pedometry on a Head-mounted Display
}

\author{
Ilias Apostolopoulos \\ University of Nevada \\ ilapostecse.unr.edu
}

\author{
Daniel S. Coming \\ Google, Inc. \\ caveman@google.com
}

\author{
Eelke Folmer \\ University of Nevada \\ efolmerecse.unr.edu
}

\begin{abstract}
The accuracy of pedometry varies depending on where an inertial sensor is located on the body. Motivated by the increasing popularity of wearable computing, this paper investigates the accuracy with which pedometry can be achieved on a head-mounted device: something previous research has not investigated. A study with 16 subjects compares the accuracy of pedometry for walking and running with an inertial sensor located at the head, pocket and hand/arm. Our study did not detect a significant difference in step counting accuracy between sensor locations, which demonstrates the feasibility of pedometry-based apps for head-mounted displays.
\end{abstract}

\section{Author Keywords}

Pedometry; Wearable computing; Activity tracking; Glass; Accelerometers; Dead reckoning; Head-mounted display

\section{ACM Classification Keywords}

H.5.m. HCI: Miscellaneous

\section{INTRODUCTION}

Wearable computing has garnered significant public interest, with a number of systems currently available. Of specific interest are smart-glass based head-mounted displays (HMD), like Google Glass. Wearables typically support eyes- and hands-free interaction, which allows them to be used in active contexts, such as walking or running. Pedometry, i.e, step counting, is used in various mobility related applications, such as physical activity tracking [13] or infrastructure-free indoor navigation [8]. Pedometry can be achieved with wearable accelerometers, e.g., Fitbit [2], or using inertial sensors that have become ubiquitous in smartphones [6] and HMDs. Because wearables are often used in active contexts, they are an especially good fit for pedometry-based applications, like physical activity tracking. The accuracy of pedometry varies depending on where an inertial sensor is placed on the human body $[11,6]$, with a higher accuracy achieved for a sensor closest to the feet [9] due to damping. The head location has not been explored and is furthest from the feet. This paper investigates whether pedometry on an HMD can be achieved with the same accuracy as when using a smartphone held at conventional locations. In addition to walking, we investigate the performance for running, as this has not been investigated and is relevant for wearable physical activity tracking apps.

Permission to make digital or hard copies of part or all of this work for personal or classroom use is granted without fee provided that copies are not made or distributed for profit or commercial advantage and that copies bear this notice and the full citation on the first page. Copyrights for third-party components of this work must be honored. For all other uses, contact the owner/author(s). Copyright is held by the author/owner(s).

CHI 2015, April 18-23, 2015, Seoul, Republic of Korea.

ACM 978-1-4503-3145-6/15/04.

http://dx.doi.org/10.1145/2702123.2702143

\section{RELATED WORK}

The accuracy of pedometry for different sensor placements has been investigated in the following work. Foster et al. [9] compared the accuracy of a waist-worn pedometer with two ankle-worn pedometers. Their results show that the latter setup offers a significantly higher accuracy but requires wearing two pedometers. Graser et al. [10] studied the accuracy of pedometers in various locations near the waistline, including navel, thigh (front/back), side (right), and back. Their study found no significant difference in accuracy between locations. A longitudinal study by Ling et al. [11] explored three different positions (bra strap, waist, shoe) for pedometers on women. Step counts from pedometers placed on the bra strap or shoe varied by more than $10 \%$ from the waist.

Though wearable pedometers are popular, they are not nearly as ubiquitous as smartphones. Brajdic \& Harle [6] evaluated the accuracy of pedometry for walking using sensors available in smartphones. Their study varied the location at which the smartphone was placed (front pant pocket, back pocket, backpack, handbag, handheld) and compared step counting algorithms. They found no significant difference in step counting accuracy between positions. However, with the phone carried in the back pocket, the likelihood of undercounting steps increases, due to extra oscillations caused by the relaxation of the gluteus maximus during the walking cycle. A related study found smartphones to be more accurate for energy expenditure prediction than wearable accelerometers [13]. Smartphones feature barometers which can be appropriated to distinguish between walking up or down stairs which generate different amounts of energy expenditure.

Specifically related to HMDs are the following work. Manohar et al. [12] demonstrated the feasibility of an earpiece with an internal accelerometer to classify activities (sitting, standing, walking). Their study did not involve pedometry, nor were comparisons made with other locations on the body. Atallah et al. [5] evaluated different locations (ear, chest, arm, wrist, waist, knee and ankle) of an accelerometer for classification of every day activities. These activities were classified by their intensity: low (eating/reading), medium (walking/vacuuming) and high level (running/cycling). Their results showed that for low level activities, a waist worn sensor yielded the the best performance. For medium level activities, the chest and wrist locations performed best. For high level activities, wearing the sensor at the ear yielded the best performance, as this location can more accurately sense the change in body posture compared to other positions. Pedometry was not evaluated. Cinaz \& Kenn [7] implemented pedometry on a head-mounted device, but no comparisons with other body locations were made. 


\section{EXPERIMENTAL SETUP}

For this experiment, we compare the accuracy of pedometry on an HMD with pedometry on a smartphone. This choice is motivated by wearable devices featuring the same inertial sensors as smartphones. HMDs and smartphones are devices that can run apps whereas wearable accelerometers only offer limited user interaction. HMDs and smartphones are also heavier than wearable accelerometers. Our experiments involve running in addition to walking, as this is a common activity for physical activity tracking, and no studies exist that evaluate the accuracy of step counting for running using smartphones. We also vary the location of the smartphone.

\section{Instrumentation}

We use Google Glass as our HMD. Google Glass is a wearable voice-controlled Android device that resembles a pair of glasses. Glass has various sensors: a camera, a microphone, 3 -axis accelerometer, 3-axis gyroscope, 3-axis magnetometer, ambient light, and a proximity sensor. Glass weighs 50 grams and runs on the Android OS. For our smartphone we used a Samsung 19300 Galaxy S III, because this phone features the same 3-axis accelerometer (Invensense MPU6050) as Google Glass, minimizing differences in results due to differences in hardware. This smartphone weights 133 grams and measures 137 × 71 × $9 \mathrm{~mm}$ and runs on Android OS. We developed a single Android 4.0.3 app to collect acceleration data. The same app ran on both the smartphone and Glass to mitigate differences in performance due to differences in code or OS. When the app is running, data collection starts as soon as the smartphone screen or the Glass touchpad is tapped. The app creates a log file for each trial with each line holding a sample and each sample containing a timestamp and the accelerations for the $\mathrm{X}, \mathrm{Y}$, and $\mathrm{Z}$ axis. If the screen or touchpad is tapped again, data collection stops. We downsampled Glass from $200 \mathrm{~Hz}$ to $100 \mathrm{~Hz}$ to match the sampling frequency of the smartphones. In order to synchronize the traces prior to each trial, each app sends a time request to a Network Time Protocol server at the beginning of each trial. The response from the server is then used as the offset from the device's native clock and is added to every timestamp we record from the sensor. Experimental results showed such time requests have an accuracy of a few tens of milliseconds, which we deemed acceptable.

\section{Participants}

We recruited 16 students ( 8 females and 8 males, average age $29.69, \mathrm{SD}=7.14$, average height $172 \mathrm{~cm}, \mathrm{SD}=9.85 \mathrm{~cm}$ ) to participate in our experiment. One subject was left handed. None of the subjects self-reported any non-correctable impairments in perception or limitations in mobility.

\section{Procedure}

Subjects were equipped with a Google Glass device. For walking, subjects held one smartphone in their dominant hand, and placed a second smartphone in their right front pant pocket. As a prior study already explored different smartphone locations for pedometry [6], we limit our study to these two most common locations; they should yield the same performance. Prior to equipping the subjects, an observer activated each app to start the data collection, first starting the smartphones and then Glass. Subjects were instructed not to touch the smartphone screens and Glass touchpad during the trial. Subjects were then asked to walk 40 steps in a straight line. We used a straight line as this was also used in previous pedometry studies $[6,13]$ and resembles the type of interaction for which accurate pedometry on an HMD offers the most useful applications (e.g., indoor navigation/health tracking). Experiments were conducted in an indoor environment: a long hallway that was approximately eight feet wide with floors made out of linoleum tiles. This environment was free of obstacles and people. Once each subject completed 40 steps, that subject tapped the touchpad on the Glass device to end the trial, and then the observer stopped the data collection on the smartphones. To gather data on running, each subject wore Glass, attached one smartphone to the bicep of their dominant arm using an armband, and placed a second smartphone in their right front pant pocket. Subjects ran 30 steps and followed the same procedure as for walking. 30 steps running was approximately the same distance as 40 steps walking. Data collection on the Glass was ended first followed by the smartphones. Subjects were asked to perform each walking and running trial three times. Subjects were split into two groups with the first group first walking and then running and the other group the other way around. Each trial was video recorded. Subjects were instructed to verbally count out their steps. An observer also counted the number of steps each subject took in each trail.

\section{RESULTS}

After the trials, the raw acceleration data from all three devices were gathered and analyzed. Each application execution represents a trace. Each entry in a trace includes accelerations in the three major axes with their corresponding timestamps. To analyze this data, the acceleration magnitude was calculated for each entry. The data was then smoothed using a centered moving average. The traces were matched with each other and synchronized based on their timestamps. As the Glass app was activated last and ended first, the start and end timestamps on the Glass trace were used to trim the smartphone traces to avoid picking up unintentional accelerations associated with touching and attaching the smartphones. A video review verified the step count for each trial.

\section{Step counting algorithm}

Brajdic \& Harle [6] evaluated the performance of various step counting algorithms on smartphones. The best results were found for windowed peak detection WPD, hidden Markov model (HMM), and continuous wavelet transform (CWT) algorithms (see [6] for references). Of these three techniques WPD is simplest to implement. WPD first smooths the acceleration magnitude using a centered moving average window of size (MovAvg). WPD detects steps using a sliding window of a fixed size (PeakDet) to detect a single peak. The algorithm finds the maximum value in the window and then shifts the window. If, after the shift, a new max is found, the previous peak is replaced if it is still present in the window. We use WPD for our experiments, as a previous study [6] found WPD to offer the same performance as more complex algorithms. WPD is computationally inexpensive, which reduces battery consumption on mobile devices. 


\begin{tabular}{|c||ccc|ccc|}
\hline \multirow{2}{*}{ Subject } & \multicolumn{3}{c|}{ Walk } & \multicolumn{3}{c|}{ Run } \\
\cline { 2 - 7 } & hand & head & pocket & arm & head & pocket \\
\hline \hline 1 & 2.5 & 3.33 & 1.67 & 2.22 & 1.11 & 3.33 \\
2 & 3.33 & 3.33 & 2.5 & 0 & 3.41 & 2.3 \\
3 & 0.81 & 5.73 & 0 & 0 & 0 & 0 \\
4 & 5 & 3.33 & 3.33 & 1.08 & 0 & 1.08 \\
5 & 0.83 & 1.67 & 4.17 & 0 & 0 & 3.23 \\
6 & 0 & 0 & 1.67 & 1.11 & 0 & 0 \\
7 & 3.33 & 0 & 0 & 1.08 & 1.08 & 1.08 \\
8 & 0.83 & 0 & 1.67 & 0 & 0 & 1.08 \\
9 & 0 & 1.67 & 1.67 & 3.41 & 0 & 1.15 \\
10 & 7.5 & 2.5 & 5 & 2.19 & 0 & 0 \\
11 & 0 & 0.83 & 0.83 & 0 & 1.08 & 1.08 \\
12 & 1.69 & 0.85 & 1.69 & 2.19 & 0 & 0 \\
13 & 0 & 2.5 & 2.5 & 0 & 0 & 2.22 \\
14 & 1.67 & 0 & 5 & 1.08 & 3.19 & 3.18 \\
15 & 2.5 & 1.67 & 2.5 & 1.08 & 0 & 1.11 \\
16 & 3.33 & 3.33 & 2.5 & 2.12 & 1.08 & 2.12 \\
\hline \hline Average & 2.08 & 1.92 & 2.29 & 1.1 & 0.68 & 1.4 \\
\hline St.Dev & 2.02 & 1.59 & 1.46 & 1.04 & 1.09 & 1.13 \\
\hline
\end{tabular}

Table 1: Step counting error (\%) for individual subjects and positions

\section{Error Extraction}

The error in our experiments is defined as the absolute difference of the number of steps calculated by the algorithm from the ground truth:

$$
e_{i j}(k)=\frac{\mid \text { steps }_{i j}(k)-\widehat{\text { steps }}_{i j}(k) \mid}{\text { steps }_{i j}(k)}
$$

where $i$ represents a specific position, $k$ is the trial, $\widehat{\text { steps }}_{i j}(k)$ is the approximation of steps subject $j$ took according to WPD, steps $s_{i j}$ is the ground truth observation for the number of steps subject $j$ took, and $e_{i j}(k)$ is the error for user $j$ for a specific position $i$, for a specific trial $k$. Each subject performed three trials for walking and running. The error for each trial is defined as:

$$
e_{i j}=\frac{\sum_{k=1}^{n} e_{i j}(k)}{n}
$$

where $e_{i j}$ represents the average error for subject $j$ at position $i$, and $n$ represents the number of trials $(n=3)$.

\section{Parameter Optimization}

Because running and walking can be accurately classified [5], we use different parameter values for walking and running to optimize the performance of the WPD algorithm. A visual inspection of traces annotated with steps detected revealed that step counting errors are either caused by: (1) the selection of the window size (PeakDet); or (2) noise from the user (MovAvg). Too much smoothing eliminates valid peaks and not enough smoothing causes errors due to noise. Though we optimized the WPD parameters, improvement of the step detection algorithm was not an objective of this research. To determine the optimum values for these parameters, an exhaustive search was performed of all reasonable values to minimize the overall average step counting error in a combined dataset for all users and positions but split by type of activity. Table 2 shows the selected values.

\begin{tabular}{|l|l|}
\hline Experiment & Parameter Values \\
\hline \hline Walking & MovAvg $=280 \mathrm{~ms}$, PeakDet $=430 \mathrm{~ms}$ \\
\hline Running & MovAvg $=175 \mathrm{~ms}$, PeakDet $=230 \mathrm{~ms}$ \\
\hline
\end{tabular}

Table 2: Optimal WPD parameter values for walking and running

Accuracy Comparison

Table 1 lists the average step counting error over the three trials for running and walking for the three positions explored. For the walking trials, the head position achieved the best results with an average error of $1.92 \%(\mathrm{SD}=1.59 \%)$. Similarly, for the running trials, the head position yielded the best results with an average error of $0.68 \%(\mathrm{SD}=1.09 \%)$. A one-way ANOVA found no statistically significant difference in average step counting error rate between head, hand and pocket for walking $\left(\boldsymbol{F}_{8,39}=.968, p=.475\right)$ or running $\left(\boldsymbol{F}_{2,45}=.269, p=.765\right)$ For walking and running, no significant difference between over, under, and exact counting of steps was found. A one-way ANOVA found a significant difference in error rate between walking and running for the head and pocket positions $\left(\boldsymbol{F}_{1,61}=16.863, p=.000\right)$. The hand and arm error rates were excluded from this analysis as these positions cannot be directly compared.

\section{DISCUSSION AND FUTURE WORK}

Our experiments found no significant difference in step counting accuracy between an HMD and smartphones worn in the user's pant pocket or held in the hand/arm. This result is contrary to what we expected. We anticipated step counting would be worse for the head location, as accelerations would be more damped, since the head is more distant from the feet than the other locations we explored. Our assumption was also corroborated by related studies $[9,11]$ that found the accuracy of step-counting to improve the closer the sensor is located to the feet. Figure 1 shows six traces of the observed acceleration for the three different locations for a running and walking trial. Though the amplitudes of the peaks for the pocket location are slightly higher than for the head and hand locations, this difference does not significantly affect the performance of the WPD algorithm.

The step counting error for running was significantly lower than for walking. When running, the heel strikes the ground with a higher velocity than when walking, so the magnitude of the observed accelerations is larger. The amplitude and frequency of the peaks are significantly higher for running than for walking. Some notable differences between traces for different locations of the accelerometer can be observed. The head (Fig. 1a \& 1d) and hand (Fig. 1b) traces resemble smooth sinusoids. The arm (Fig. 1e) and pocket (Fig. $1 \mathrm{c} \& 1 \mathrm{f}$ ) traces look different and resemble unbalanced sinusoids with peaks that alternate in amplitude, i.e. a high peak is followed by a low peak. Because the pocket and arm locations are right above the leg, they pick up accelerations from a heel strike from one leg more strongly than the other. The closer an accelerometer is placed to the sagittal plane, i.e., the vertical plane dividing the body into a left and right half, the more balanced the sinusoid of the acceleration signal will be. More accurate pedometry algorithms could potentially be developed that exploit the asymmetrical inertial signals of the pocket and arm locations. 


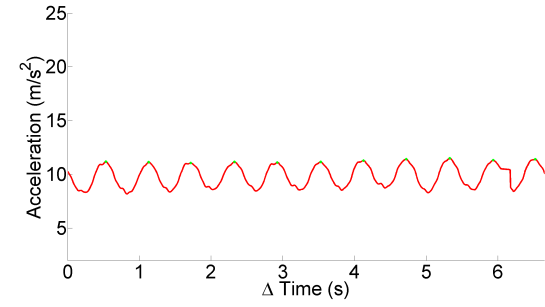

(a) Walking: Head

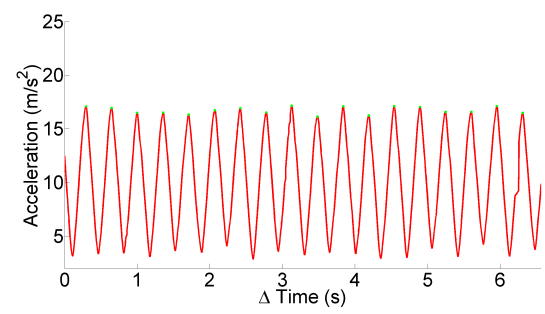

(d) Running: Head

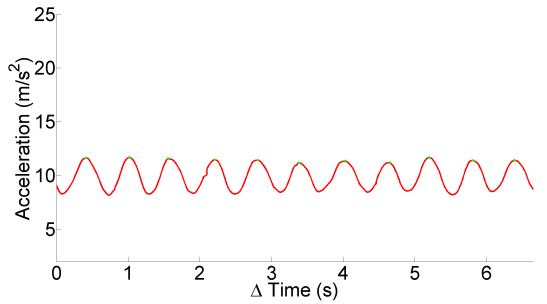

(b) Walking: Hand

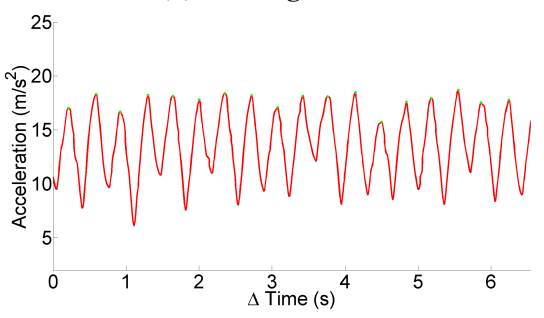

(e) Running: Arm

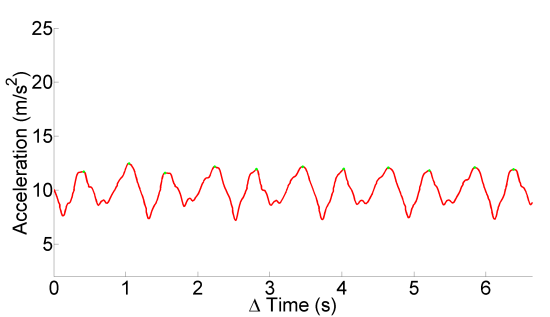

(c) Walking: Pocket

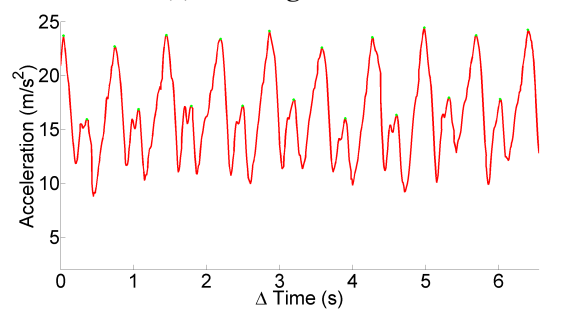

(f) Running: Pocket

Figure 1: Sample traces of acceleration magnitudes for a walking and running trial. The top traces are for walking with locations: a) head, b) hand, c) pocket. The bottom traces are for running with locations: d) head, e) arm, f) pocket.

For running, the arm picks up additional accelerations from swinging the arms back and forth, which causes the amplitudes of these peaks to be slightly higher than for the head location (see Fig.1e \& 1d). Though no significant difference between locations was found, the step counting error for running for the hip and bicep were nearly twice that of the head. Smart watches are considered less obtrusive than smart glasses [4] but this result eludes to that pedometry on a smart glasses may be more accurate than on a smart watch given that runners swing their arms while running and a higher noise can be expected for the wrist position than the bicep position.

The Samsung smartphone weighs nearly three times as much as Glass. This difference in mass could affect its ability to accurately pick up accelerations (with heavier devices sensing accelerations more accurately). The larger distance from the feet makes it harder for Glass to pick up accelerations, and noise is more falsely classified as a step. However, Glass is pretty firmly attached to the user's head as it rests on the nose bone where the skin is very thin, which minimizes damping. A human head also weighs approximately $8 \%$ of the total body weight, which overall leads to relatively smooth sinusoids with little noise that allow for accurate step detection.

Currently, wearable devices are tethered to smartphones for their mobile data feed, though it is possible to use Glass as a standalone device in areas with WiFi. Recently, standalone smart watches (e.g. Samsung Gear S[3]) have been introduced, and smart glasses could become standalone devices eventually. Results of this research demonstrate the feasibility of pedometry on smart-glass based HMDs. This enables useful applications such as infrastructure-less indoor navigation and physical activity tracking that take advantage of hands-free input and always available feedback (e.g. step count or calorie expenditure). Our results may also apply to "hearables", i.e., standalone smart in-ear headphones (e.g., Bragi The Dash [1]) that offer fitness tracking functionality.

\section{ACKNOWLEDGEMENTS}

This work is supported by a Google Faculty Research Award.

\section{REFERENCES}

1. Bragi The Dash, bragi.com.

2. Fitbit wearable activity trackers, fitbit.com.

3. Samsung Gear S, samsung. com/gears.

4. Amadeo, R. Android Wear smartwatches make Google Glass obsolete, In Ars Technica,'14.

5. Atallah, L., et al. Sensor placement for activity detection using wearable accelerometers. In Proc. of BSN '10, 24-29.

6. Brajdic, A., and Harle, R. Walk detection and step counting on unconstrained smartphones. In Proc. of UbiComp '13, 225-234.

7. Cinaz, B., and Kenn, H. Headslam - simultaneous localization and mapping with head-mounted inertial and laser range sensors. In Proc. of ISWC '08, 3-10.

8. Fallah, N., et al. The user as a sensor: navigating users with visual impairments in indoor spaces using tactile landmarks. In Proc. of CHI' 12, 425-432.

9. Foster, R. C., et al. Precision and accuracy of an ankle-worn accelerometer-based pedometer in step counting and energy expenditure. Prev Med 41, 3-4 ('05), 778-83.

10. Graser, et al. Effects of placement, attachment, and weight classification on pedometer accuracy. J Phys Act Health 4, 4 (10/'07), 359-69.

11. Ling, C. G., and Smith, S. O. Alternative placements for women wearing pedometers. J Am Acad Nurse Pract 22, 5 (5/'10), 264-9.

12. Manohar, et al. An accelerometer-based earpiece to monitor and quantify physical activity. $J$ Phys Act Health 6, 6 (11/09), 781-9.

13. Pande, A., et al. Energy expenditure estimation with smartphone body sensors. In Proc. of BodyNets'13, 8-14. 